INVESTIGACIÓN

\title{
La aceptación de la enfermedad y la adhesión al tratamiento en pacientes con epilepsia
}

\author{
The acceptance of disease and the treatment adherence in the \\ patient with epilepsy
}

\author{
Justo R. Fabelo Roche, ${ }^{\text {I }}$ Libertad Martín Alfonso, ${ }^{\text {II }}$ Serguei Iglesias Moré ${ }^{I I I}$ \\ ILicenciado en Psicología. Doctor en Ciencias de la Salud. Universidad de Ciencias \\ Médicas de La Habana, Cuba. \\ II Licenciada en Psicología. Doctor en Ciencias de la Salud. Escuela Nacional de Salud \\ Pública. La Habana, Cuba. \\ IIILicenciado en Enfermería. Máster en Psicología de la Salud. Universidad de \\ Ciencias Médicas de La Habana, Cuba.
}

\section{RESUMEN}

Introducción En la consulta especializada de epilepsia del Hospital Psiquiátrico de la Habana se identificaron dificultades para lograr adhesión al tratamiento en pacientes con diagnóstico clínico de epilepsia. Los pacientes no aceptan la enfermedad que padecen, se agotan del carácter continuo del tratamiento y no están dispuestos a asumir los ajustes que demanda su estilo de vida.

Objetivo Evaluar la efectividad de una estrategia psicoterapéutica y educativa dirigida a potenciar la aceptación de la enfermedad y a mejorar el nivel de adhesión terapéutica.

Métodos La citada estrategia incluyó sesiones dirigidas a promover el desarrollo de una actitud responsable ante la enfermedad y a la asimilación de las limitaciones que impone. Incluyó recursos racionales, vivenciales y educativos para reforzar la aceptación de la enfermedad y la adhesión terapéutica. La tarea básica inicial fue identificar y modificar la tendencia a ocultar ante los demás y ante sí mismo que padecen de epilepsia.

Resultados El nivel promedio obtenido en la escala de adaptación a la medicación y al tratamiento mejoró a partir de la intervención desarrollada. De un puntaje medio de 2,23 que sugiere la presencia de problemas en la adaptación psicosocial a la medicación se pasó a un puntaje medio 1,09 que expresa la ausencia de problemas significativos en esta área.

Conclusiones La estrategia psicoterapéutica y educativa dirigida a potenciar la

http://scielo.sld.cu 
aceptación de la enfermedad y a mejorar el nivel de adhesión terapéutica es efectiva.

Palabras clave: Epilepsia, aceptación de la enfermedad, adhesión terapéutica, adaptación a la medicación y al tratamiento medico.

\section{ABSTRACT}

Introduction The specialized medical service for epilepsy in the psychiatric hospital of Havana identified several difficulties to succeed in making the patient diagnosed with epilepsy follow the corresponding treatment. The patients do not accept their disease; they get tired of the continued therapy, and refuse to make the necessary arrangements in their lifestyle.

Objectives To evaluate the effectiveness of the application of a psychotherapeutic and educational strategy aimed at encouraging acceptance of disease and improving treatment adherence.

Methods This strategy included sessions to develop responsible attitude toward the disease and to accept the restrictions that it imposes upon the patient. It also covered rational, affective and educational resources. The initial basic therapeutic task was to identify and to change the tendency of the patient to hide his/her epilepsy from himself/herself and from the others.

Results The average scoring in the medication and treatment adherence scale improved after this intervention. From an average score of 2.23, indicating problems in psychosocial adaptation to medication, to 1.09 expressing lack of significant problems in this area.

Conclusions The psychotherapeutic and education strategy to encourage the acceptance of disease by the patient and to improve treatment adherence was effective indeed.

Key words: Epilepsy, acceptance of disease, therapeutic adherence, adaptation to medication and to medical treatment.

\section{INTRODUCCIÓN}

La epilepsia es una enfermedad de origen orgánico cuya causa fundamental reside en la actividad eléctrica anormal del cerebro. Se trata de una afección crónica de patogenia diversa, caracterizada por la presencia de crisis eléctricas recurrentes, debidas a una descarga excesiva de las neuronas cerebrales. Eventualmente pueden estar asociadas con variadas manifestaciones clínicas y paraclínicas. ${ }^{1}$

La severidad de sus manifestaciones agudas está asociada a la presencia de tres grupos fundamentales de factores de riesgo: ${ }^{2}$

1. Factores neurobiológicos: edad de inicio de las crisis y su duración, tipos de ataques y su control, características electroencefalográficas, presencia de daño cerebral, metabolismo cerebral y neurotransmisores.

http://scielo.sld.cu 
2. Factores psicosociales: temor a las crisis, calidad de vida deficiente, problemas financieros, escasa disponibilidad de apoyo social y estigmas sociales y familiares.

3. Factores medicamentosos: politerapia anticonvulsivante, tratamiento con barbitúricos, déficit de folatos y efecto hormonal.

El enfoque clínico tradicional en el tratamiento de la epilepsia enfatiza los aspectos neurobiológicos y medicamentosos. Ante un paciente con epilepsia los esfuerzos se centran en el control de las crisis y para ello no se escatiman recursos técnicos novedosos y fármacos anticonvulsivantes variados de demostrada efectividad. ${ }^{3}$ De esta forma, es posible el control del fenómeno ictal en alrededor del $80 \%$ de los casos, queda el $20 \%$ de las llamadas epilepsias refractarias al tratamiento médico para las cuales se emplean otros recursos terapéuticos como la cirugía y la medicina verde, entre otros. ${ }^{4}$

Esta posición subraya la condición "enfermedad" y olvida que estos enfermos enfrentan una complicada situación psicosocial. La epilepsia es una afección estigmatizante que desde tiempos inmemoriales se vinculó a elementos sobrenaturales y que en consecuencia ha generado prejuicios, subvaloraciónes y conceptos erróneos. ${ }^{5}$ Ello determina que muchos de estos seres humanos sufran desde sobreprotección durante la niñez que impide el desenvolvimiento de sus potencialidades y el desarrollo de la personalidad, hasta discriminación injustificada para desempeñarse en determinados empleos en la edad adulta.

Son comunes en estos enfermos trastornos psicológicos que afectan su bienestar general y que son responsables de no pocas recaídas y descompensaciones de sus crisis, aun cuando se mantenga estable la medicación anticonvulsivante. Un precario desarrollo de la autoestima y la autoconfianza, una significativa tendencia a la depresión y la ansiedad, un limitado manejo del estrés por la carencia de habilidades de afrontamiento, un bajo nivel de adhesión terapéutica, los temores y la inseguridad, la insatisfacción vital y otros, introducen limitaciones y sesgos en el éxito del tratamiento clínico tradicional. ${ }^{6}$

Actualmente adquiere mayor significación el diseño de programas de rehabilitación útiles para mejorar los índices de adaptación social en estos pacientes. Proveer facilidades, no solo médicas, sino también psicosociales, es necesario para asegurar un mejor afrontamiento de los pacientes a las dificultades a las que se ven expuestos. La rehabilitación requiere de un conjunto de medidas que van más allá del adecuado uso de los medicamentos para cada tipo de crisis. Es necesario crear instancias sociales que permitan la recuperación integral del paciente con epilepsia bajo un modelo multietiológico que incluya los diferentes aspectos comprometidos en la enfermedad ya sea biológicos, psicológicos o sociales. ${ }^{7}$

Partimos del criterio de que eliminar las crisis no asegura que el paciente erradique las dificultades que esta enfermedad le impone. Aunque siempre el imperativo médico-terapéutico al tratar al paciente con epilepsia es el control de las crisis, para quienes nos aproximamos a este problema desde las Ciencias de la Salud, si bien es importante controlar estas crisis, también es muy importante lograr una adaptación psicosocial efectiva del paciente, eliminar los estigmas sociales y familiares, mejorar la calidad de vida y contribuir al bienestar y la felicidad de esas personas.

La Epileptología es una ciencia multidisciplinaria emergente que integra a los diversos profesionales de la salud que proveen una atención multilateral al

http://scielo.sld.cu 
paciente. La propia Liga Internacional contra la Epilepsia ha reconocido que la intervención de la psicología de la salud es útil para promover la comprensión de la enfermedad entre los pacientes y para puntualizar el diagnóstico y el tratamiento; además de considerarla indispensable para la evaluación prequirúrgica y para la rehabilitación del paciente con epilepsia. ${ }^{8}$

Sin embargo, la práctica asistencial e investigativa permite identificar un conjunto de limitaciones en la atención integral al paciente con epilepsia en todos los niveles de atención: un pensamiento profesional muy centrado en el individuo enfermo y en el método clínico individual, pobre consideración de los factores psicosociales que afectan a estos pacientes, insuficiencias en los conocimientos básicos que sobre la temática necesitan los profesionales encargados de su atención, carencia de una bibliografía apropiada para el estudio del tema y dispersión de la existente y déficit de modelos conceptuales y procedimientos psicoterapéuticos aplicables al contexto de la atención primaria de salud (APS).

Una aproximación exploratoria a este problema en el contexto de la Consulta Especializada de Epileptología del Hospital Psiquiátrico de la Habana (HPH) nos permitió identificar la existencia de dificultades significativas para lograr adhesión al tratamiento en pacientes con diagnóstico de epilepsia. Por lo general, el paciente se niega a aceptar la enfermedad que padece, se agota del carácter continuo del tratamiento y no está dispuesto a asumir ajustes en su estilo de vida.

\section{MÉTODOS}

Se realizó un estudio explicativo de corte longitudinal con el objetivo de evaluar la efectividad de la aplicación de una estrategia psicoterapéutica y educativa dirigida a potenciar la aceptación de la enfermedad como condición intrínseca de la vida y en función de ello lograr una adecuada adhesión al tratamiento en un grupo de pacientes con diagnóstico clínico de epilepsia.

La investigación se implementó a partir de un diseño cuasi experimental, desarrollado en una muestra de 200 pacientes seleccionados aleatoriamente (tabla de números aleatorios) en la consulta especializada en epileptología del HPH.

Como pre y posprueba (antes y después de la intervención) se aplicó la Escala de Medicación y Tratamiento Médico del Washington Psichosocial Seizures Inventory (WPSI). Este instrumento fue validado para la población cubana en el 2005, para lo cual se precisó su validez de contenido a partir de criterios de expertos y se estudió su consistencia interna mediante la utilización del índice alfa de Cronbach. La estimación de la confiabilidad total del instrumento arrojó un valor de 0,84 que implica una elevada consistencia interna. ${ }^{6}$

La variable explicativa considerada fue la aplicación de las técnicas psicoterapéuticas y educativas dirigidas a fomentar la aceptación de la enfermedad y la adhesión terapéutica, mientras que la variable de respuesta fue el nivel de adaptación psicosocial a la medicación y tratamiento médico calculado a partir de la escala aplicada. Las variables de control consideradas fueron la edad, el sexo, la presencia de lesión cortical, la frecuencia de los ataques, la clasificación de las crisis y el tipo de tratamiento farmacológico utilizado.

La escala de Medicación y Tratamiento Médico del WPSI que se presenta a continuación consta de ocho reactivos y se considera una medida objetiva del

http://scielo.sld.cu 
ajuste alcanzado por estos pacientes en esta área. El sujeto debe contestar cada pregunta en forma afirmativa o negativa y su aplicación es completada en pocos minutos. La complejidad del WPSI en general es compatible con las eventuales limitaciones neuropsicológicas de los pacientes con epilepsia (recuadro).

Recuadro. Escala de Adaptación a la Medicación y al Tratamiento Médico. (Medicine and Medical Management) del WPSI

\begin{tabular}{|c|c|c|c|}
\hline No. & Preguntas & 0 & 1 \\
\hline 1 & ¿Su médico conoce plenamente todos sus problemas de salud? & Sí & No \\
\hline 2 & ¿Piensa que su médico verdaderamente se interesa por usted? & Sí & No \\
\hline 3 & ¿Se siente completamente satisfecho con su médico? & Sí & No \\
\hline 4 & ¿Se le olvida con frecuencia tomar su medicina? & Sí & No \\
\hline 5 & ¿Se siente a menudo inquieto? & Sí & No \\
\hline 6 & ¿Le agrada la forma en que lo atiende su médico? & Sí & No \\
\hline 7 & ¿Siente que sus ataques están siendo controlados lo mejor posible? & Sí & No \\
\hline 8 & $\begin{array}{l}\text { ¿Constantemente los medicamentos que toma le producen problemas } \\
\text { para dormir? }\end{array}$ & Sí & No \\
\hline
\end{tabular}

A partir de su aplicación individual se establecen cuatro niveles de adaptación psicosocial que se delimitan a partir de los puntajes alcanzados en los siguientes rangos de valores:

- Nivel 1. Sin problemas significativos: promedio de 0,00 a 1,80 .

- Nivel 2. Con problemas de limitado significado: promedio de 1,81 a 3,20.

- Nivel 3. Con problemas de claro impacto: promedio de 3,21 a 6,70.

- Nivel 4. Con problemas de grave impacto: promedio de 6,71 a 8,00.

Para la evaluación de los resultados de esta investigación se partió del cálculo de la media aritmética y de la desviación típica de la Escala de adaptación psicosocial a la medicación y al tratamiento médico del WPSI. Este instrumento se construyó en sentido ascendente, utilizando la misma polaridad para todas las preguntas, es decir que los puntajes inferiores para cada escala expresan un mejor nivel de adaptación psicosocial mientras que en la medida que los puntajes aumentan se incrementa también el nivel de inadaptación.

En la intervención psicoeducativa desarrollada se ejecutaron seis sesiones dirigidas a promover el desarrollo de una actitud responsable ante la enfermedad y para la asimilación de las limitaciones que como secuela esta impone. Incluyó recursos racionales, vivenciales y educativos para potenciar la aceptación de la enfermedad y la adhesión terapéutica.

Se realizó una sesión introductoria de encuentro con el paciente dirigida a identificar el problema psicosocial que para el paciente constituye su enfermedad, especial énfasis se hizo en la aplicación de técnicas cognitivo-conductuales y afectivo-vivenciales como la reestructuración cognitiva, la solución de problemas, la clarificación de valores y el control de la ira. Finalmente, se desarrolló una sesión de cierre en la que se valoró en que medida se había modificado el nivel de aceptación de la enfermedad y cómo se había estimulado la mejoría en la adhesión al tratamiento.

http://scielo.sld.cu 
La tarea terapéutica básica inicial fue identificar y modificar la tendencia a ocultar ante los demás y ante sí mismo que padecen de epilepsia. Si se parte de promover estilos de vida adecuados o facilitadores de la adaptación psicosocial del paciente con epilepsia, de generar ambientes positivos y de prevenir las descompensaciones que pueden deteriorar la vida, no hay razón para asumir la enfermedad de manera inconsciente y descuidar el tratamiento médico y otras posibilidades de enriquecimiento espiritual.

\section{RESULTADOS}

Al evaluar los resultados del estudio realizado se encontró que el nivel promedio de adaptación psicosocial a la medicación y al tratamiento médico mejoró a partir de la intervención desarrollada. De un puntaje medio de 2,23 que sugiere la presencia de problemas de limitado significado se pasó a un puntaje medio 1,09 que expresa la ausencia de problemas significativos en esta área.

Como puede apreciarse en la tabla que se presenta resulta evidente que los pacientes estudiados obtuvieron mejores resultados una vez aplicada la estrategia de intervención diseñada.

Tabla. Niveles de adaptación psicosocial a la medicación y al tratamiento médico obtenidos por los pacientes estudiados

\begin{tabular}{|l|l|l|l|l|l|}
\hline Escala & Evaluación & $N$ & Media & Desv. típica \\
\hline \multirow{2}{*}{ Medicación y tratamiento médico } & Inicial (preprueba) & 200 & 2,23 & 0,37 \\
\cline { 3 - 5 } & Final (posprueba) & 200 & 1,09 & 1,08 \\
\hline
\end{tabular}

En la evaluación final de los resultados de este estudio se tomaron en cuenta además otros datos obtenidos a partir de la aplicación de la técnica cualitativa de la entrevista en profundidad. Ello resultó ser de gran utilidad pues permitió complementar la información obtenida para corroborar la autenticidad del nivel de adaptación alcanzado.

El análisis cualitativo de los resultados incluyó el análisis de contenido de los datos obtenidos. La categoría "Valoración positiva del proceder médico actual" fue expresada por el $85 \%$ de los pacientes estudiados resultando la que más alto valor obtuvo en este estudio. Esta categoría se refiere a la expresión de argumentos de rechazo a tratamientos anteriores que le habían sido indicados en otros servicios, a la forma superficial con que fueron atendidos, sin explicaciones claras y errores en algunas indicaciones.

\section{DISCUSIÓN}

La medicación y tratamiento médico no parece ser un área especialmente conflictiva para los pacientes con epilepsia. A ello contribuye de manera significativa el hecho de contar con una política de salud a escala nacional que garantiza la atención a todos los enfermos, además de enfatizar en el aspecto preventivo. Evidentemente los resultados del grupo de pacientes estudiados son positivos. En ese caso se obtiene una media de 1,09 (sin problemas de adaptación

http://scielo.sld.cu 
psicosocial), mientras que ese mismo grupo de pacientes antes de la intervención había obtenido una media de 2,23 la que clasifica como "con problemas de limitado impacto en la adaptación psicosocial del paciente."

La accesibilidad de los pacientes a los servicios de salud repercute favorablemente en su adaptación psicosocial. Es cierto que existe algún nivel de desinformación en el personal médico y paramédico en relación con esta enfermedad y que esos problemas de limitado impacto detectados pueden estar en relación con ello. Precisamente, el éxito de la intervención desarrollada sugiere promover la optimización de los servicios mediante el desarrollo de actividades de capacitación y superación profesional.

A este resultado también contribuye el hecho de que en el equipo de salud que atendió a los pacientes existe un buen nivel de información acerca de la enfermedad y en consecuencia un mejor nivel de atención. Recordemos que esta escala aporta información sobre la perspectiva que tiene el paciente acerca de la atención profesional, sanitaria y farmacológica que recibe. En este sentido intenta determinar la percepción del sujeto respecto al tratamiento recibido, así como la relación médico-paciente.

El equipo multidisciplinario de epileptología del HPH, tiene establecido como norma que en la consulta el médico debe dar al paciente y a sus familiares una explicación detallada de su enfermedad, con lenguaje claro y sencillo, además de alertarlo de que no debe suspender su tratamiento con drogas antiepilépticas. ${ }^{9}$

También debe orientar sobre los factores de riesgo que suelen precipitar las crisis: la privación del sueño, la fatiga excesiva y la exposición a ambientes de potente estimulación visual o auditiva. Como ejemplo de este tipo de riesgo se reconocen entre los jóvenes las continuas noches sin dormir participando en vídeo-juegos con gran estimulación visual o en las discotecas donde se mezcla el consumo de alcohol, la privación del sueño y el efecto de las luces. No obstante, el médico debe tener cuidado con las restricciones que sugiere ya que de ser exageradas pueden contribuir a potenciar la expresión de manifestaciones depresivas.

El comportamiento y los modos de afrontamiento de las personas a la enfermedad pueden desempeñar un papel importante en su curso y, en este contexto, el hecho de que un paciente cumpla o no con las prescripciones médicas juega un papel primordial. Podrán alcanzar un mejor control de la enfermedad e incrementar o preservar su calidad de vida aquellas personas que logren adherirse adecuadamente a los tratamientos y regímenes conductuales que cada una de las enfermedades exige para su buena evolución. ${ }^{10,11}$ En la epilepsia estos criterios cobran especial relevancia. ${ }^{12}$

En la investigación se constató que la adaptación a la medicación y al tratamiento médico es un aspecto que esencialmente se favorece con la intervención y que también se mejora la adhesión al tratamiento. En este caso, se promueve una mayor participación del paciente en la toma de decisiones que afectan a su propia salud. Se supone que el paciente se adhiere a un plan con el que está de acuerdo y en cuya elaboración ha podido contribuir o, al menos, que ha aceptado la importancia de realizar acciones concretas que se incluyen en el programa a poner en práctica. ${ }^{13}$ Ello implica una consideración activa de la persona, lo cual es precisamente lo que se intenta conformar a partir de la aplicación de la estrategia de intervención.

Por otra parte, algunos ítems de la escala, los que de manera global contribuyen a disminuir el puntaje general obtenido, demuestran mejorías en la adhesión al

http://scielo.sld.cu 
tratamiento: los pacientes olvidan menos tomar su medicación, los ataques o crisis están más controladas, así como la percepción de que les agrada la forma en que los atiende su médico. De esta manera, se puede inferir que la dimensión de la adhesión de relación transaccional entre el equipo de salud y el paciente está favorecida e incide en la mejor ejecución de las indicaciones médicas. ${ }^{14}$

La categoría cualitativa "Valoración positiva del proceder médico actual" constituyó el dato de mayor relevancia asociado al indicador "Medicación y tratamiento médico", y fue manifestado por el $85,0 \%$ de los pacientes estudiados. En el análisis de la entrevista en profundidad aparecían frecuentes alusiones a múltiples tratamientos anteriores sin efectivo control de las crisis y de las alteraciones psíquicas asociadas y a quejas por orientaciones imprecisas que en la mayor parte de los casos responden a un limitado conocimiento de la enfermedad y su terapéutica en el personal médico que los atendía. Sin embargo, los pacientes tienden a evaluar este indicador con base en su valoración del tratamiento que reciben actualmente. En ese sentido manifiestan "siento que ahora sí han logrado controlar mis ataques", "nunca antes los médicos dieron importancia a mis criterios", "he aprendido como manejar esta enfermedad."

En resumen, se puede concluir que la estrategia psicoterapéutica y educativa dirigida a potenciar la aceptación de la enfermedad y a mejorar el nivel de adhesión terapéutica es efectiva.

\section{REFERENCIAS BIBLIOGRÁFICAS}

1. Gastaud H. Diccionario de epilepsia. Ginebra: OMS;1976.

2. Fabelo JR. Psicología de la Epilepsia. La Habana: Científico-Técnica; 2004.

3. Covanis A. Advances in the treatment of the epilepsies. Internal Epilepsy News. $2000 ; 139: 10-1$.

4. Lechtenberg R. La epilepsia y la familia. Barcelona: Editorial Herder; 1989.

5. Stuttaford T. Wordwide batle against the stigma of epilepsy. TheTimes (London). 22 May 1997.

6. Fabelo JR. Paradigma psicológico salubrista para la atención al paciente con epilepsia. La Habana: Editorial Universitaria del Ministerio de Educación Superior; 2008.

7. Ivanovic-Zuvic F, Alvarado L. Evaluación psicosocial de los epilépticos en Chile. Rev Chilena Neuropsiquiatría. 2001;39(4):303-15.

8. World Health Organization. Professionals allied to medicine. Epilepsy Atlas. 2005:52-3.

9. González S, Quintana J, Fabelo JR. Epilepsia y sociedad: una mirada hacia el Siglo XXI. Rev Psiquiatría.com [serie en Internet]. 1999 [citado 1 Oct 2005];3(3). Disponible en: http://www.psiquiatria.com/psiquiatria/revista/vol3num3/

10. Ferrer VA. "Adherencia a" o "cumplimiento de" prescripciones terapéuticas y de salud: concepto y factores psicosociales implicados. J Health Psichol. 1995;7(1).

http://scielo.sld.cu 
11. Martín L. Acerca del concepto de adherencia terapéutica. Rev Cubana Salud Pública [serie en Internet]. 2004 Dic [citado 29 Sep 2010];30(4). Disponible en: http://scielo.sld.cu/scielo.php?script=sci arttext\&pid=S086434662004000400008\&lng=es

12. McAuley JW, Hollis IB, Wolfe T. An evaluation of medication adherence and self management techniques in patients with epilepsy. Epilepsia. 2005;46(supl 8):349.

13. Martín L, Grau J. La investigación de la adherencia terapéutica como un problema de la psicología de la salud. Psicología y Salud. 2004;14(1): 75.

14. Martín L. Adherencia al tratamiento en hipertensos de áreas de salud del nivel primario [tesis]. La Habana: Escuela Nacional de Salud Pública; 2009.

Recibido: 1 de febrero de 2010.

Aprobado: 12 de mayo de 2010.

Justo R. Fabelo Roche. Calle 146 No. 2504 e/ 25 y 31, Cubanacán 11600, Playa. La Habana, Cuba.

Correo electrónico: fabelo@infomed.sld.cu 